\title{
VISCOSITY SOLUTIONS METHODS FOR CONVERSE KAM THEORY*
}

\author{
Diogo A. Gomes ${ }^{1}$ And Adam OBerman ${ }^{1}$
}

\begin{abstract}
The main objective of this paper is to prove new necessary conditions to the existence of KAM tori. To do so, we develop a set of explicit a-priori estimates for smooth solutions of HamiltonJacobi equations, using a combination of methods from viscosity solutions, KAM and Aubry-Mather theories. These estimates are valid in any space dimension, and can be checked numerically to detect gaps between KAM tori and Aubry-Mather sets. We apply these results to detect non-integrable regions in several examples such as a forced pendulum, two coupled penduli, and the double pendulum.
\end{abstract}

Mathematics Subject Classification. 37J50, 49L25, 65P10, $70 \mathrm{H} 7$.

Received December 26, 2006. Revised March 23, 2008.

Published online September 25, 2008.

\section{INTRODUCTION}

Let $H: \mathbb{R}^{n} \times \mathbb{R}^{n} \rightarrow \mathbb{R}, H(p, x)$, be the Hamiltonian of a mechanical system. A classical procedure to determine solutions to the Hamiltonian dynamics

$$
\dot{\mathbf{p}}=D_{x} H(\mathbf{p}, \mathbf{x}), \quad \dot{\mathbf{x}}=-D_{p} H(\mathbf{p}, \mathbf{x}),
$$

is the Hamilton-Jacobi method $[1,18]$. This method has two main steps. The first one consists in computing a pair of functions $(u(x, P), \bar{H}(P)), u: \mathbb{R}^{n} \times \mathbb{R}^{n} \rightarrow \mathbb{R}$, and $\bar{H}: \mathbb{R}^{n} \rightarrow \mathbb{R}$, which solves the Hamilton-Jacobi equation:

$$
H\left(P+D_{x} u, x\right)=\bar{H}(P)
$$

the second step is the construction of a change of coordinates $X(p, x)$ and $P(p, x)$, defined implicitly through the equations

$$
p=P+D_{x} u, \quad X=x+D_{P} u .
$$

In these new coordinates, the Hamiltonian dynamics becomes:

$$
\dot{\mathbf{P}}=0, \quad \dot{\mathbf{X}}=-D_{P} \bar{H}(\mathbf{P}) .
$$

Thus, for each $P$ the graph

$$
p=P+D_{x} u(x, P),
$$

is invariant under the flow generated by (1.1). Furthermore, the flow on this graph is conjugated to a translation as $\dot{\mathbf{X}}$ is constant.

Keywords and phrases. Aubry-Mather theory, Hamilton-Jacobi integrability, viscosity solutions.

* D. Gomes was partially supported by CAMGSD/IST through FCT Program POCTI FEDER and by grant POCI/FEDER/ MAT/55745/2004, DENO/FCT-PT (PTDC/EEA-ACR/67020/2006).

${ }^{1}$ Instituto Superior Tecnico, Department of Mathematics, Av. Rovisco Pais, 1049-001 Lisboa, Portugal.

dgomes@math. ist.utl.pt 
If the Hamiltonian $H(p, x)$ is $\mathbb{Z}^{n}$ periodic in $x$, and $u(x, P)$ is a $\mathbb{Z}^{n}$ periodic function in $x$, that is, $H(p, x+k)=$ $H(p, x)$, and $u(x+k)=u(x)$, for all $p, x \in \mathbb{R}^{n}$, and $k \in \mathbb{Z}^{n}$, the graph (1.5) can be interpreted as an invariant torus. In this periodic case, it is natural to regard $H$ as a function $H: \mathbb{T}^{n} \times \mathbb{R}^{n} \rightarrow \mathbb{R}$, where $\mathbb{T}^{n}=\mathbb{R}^{n} / \mathbb{Z}^{n}$ is the $n$ dimensional torus. In (most of) this paper we assume further that the Hamiltonian $H(p, x): \mathbb{T}^{n} \times \mathbb{R}^{n} \rightarrow \mathbb{R}$ is smooth and strictly convex in $p$, that is, $D_{p p}^{2} H>\gamma>0$.

It is well known that the Hamilton-Jacobi method may fail in practice. Indeed, (1.2) may not admit smooth solutions, or (1.3) may not define a smooth change of coordinates. In particular, the Hamilton-Jacobi method may be valid for certain initial conditions of (1.1) but not everywhere.

The main question we would like to address in this paper is the following: given initial conditions $(p, x)$ for (1.1), can this integrability procedure be carried out? More precisely, is there a vector $P$, and a pair $(u, \bar{H})$ solving (1.2) such that $p=P+D_{x} u(x)$ ? In the case in which the Hamiltonian $H$ has the special structure

$$
H(p, x)=H_{0}(p)+\epsilon H_{1}(p, x),
$$

one can apply the KAM theorem [1]. This theorem asserts that it is possible to use the classical Hamilton-Jacobi method for a large set of initial conditions as long as $H_{0}$ satisfies the non-degeneracy condition $\operatorname{det} D_{p p}^{2} H \neq 0$ and $\epsilon$ is sufficiently small.

Using viscosity solutions (for the general theory of viscosity solution we suggest the references $[3,7,15]$ ), one can prove that a weak form of integrability still holds, see, for instance, $[2,6,8-13,19]$, or [20]. As demonstrated in context of homogenization of Hamilton-Jacobi equations, in the classic but unpublished paper by Lions et al. [27], equation (1.2) admits viscosity solutions. We recall that a function $u$ is a viscosity solution of (1.2) if for any smooth function $\phi(x)$ and any point $x_{0}$ such that $u-\phi$ has a maximum (resp. minimum) at $x_{0}$

$$
H\left(P+D_{x} u\left(x_{0}\right), x_{0}\right) \leq \bar{H}(P) \quad(\text { resp. } \geq \bar{H}(P)) .
$$

Theorem 1.1 (Lions et al. [27]). For each $P$ there exists a unique number $\bar{H}(P)$ and a function $u(x, P), \mathbb{Z}^{n}$ periodic in $x$, that solves $(1.2)$ in the viscosity sense. Furthermore $\bar{H}(P)$ is convex in $P$ and $u(x, P)$ is Lipschitz in $x$.

Also, one can construct an invariant set contained in the graph

$$
\left(x, P+D_{x} u(x)\right) .
$$

The support of the Aubry-Mather measures [31-35], which are the natural generalizations of invariant tori, is contained in this graph. Unfortunately, not every point is in the support of a Mather measure. Heuristically, one can think of the phase space as containing several sets: the set of all KAM torus, the supports of all Mather measures, heteroclinic and homoclinic connections between different components of Mather sets, and then a remaining part of the phase space which may contain elliptic periodic orbits and corresponding elliptic islands, areas in which the motion is irregular, possibly chaotic, and regions in which Arnold diffusion may occur. It is therefore of interest to study the existence of gaps in the Mather sets, which would prevent the Hamilton-Jacobi integrability and allow for more complex dynamics. There have been several attempt to study this problem, for instance, $[24,25,28-30]$.

The approach in [29], as well as in [25,28,30], uses the well known fact that the Mather set is a Lipschitz graph - thus by detecting orbits that do not lie on a Lipschitz graph one proves the existence of gaps in the Mather set. These methods seem to work well for one-dimensional maps, but do not extend easily for multi-dimensional problems. The paper [24], studies the discrete case, and uses the fact that orbits on the Mather set, being global minimizers, are also local minimizers. Therefore, with second order tests for critical points, one can show that certain orbits lie outside the Mather set. The main advantage is that this method works for maps in any dimension. In this paper we also explore the minimizing character of the orbits in the Mather set to obtain certain inequalities which can be checked numerically. Our method works in any space dimension and, when 
compared with the one in [24], is of a global nature as it checks whether the orbits are global minimizers, not just local minimizers.

Other approaches to prove non-integrability include, among others, renormalization methods, anti-integrable limit. These methods are fundamentally different from the ones considered in this paper. Also, we should mention that there are several related papers which use variational methods to prove analytic counterexamples to KAM theory, such as $[4,16]$, and [23]. In [4,16] certain non-minimizing orbits were constructed. Measures supported in the gaps of Aubry-Mather sets were constructed in [17]. In the paper [23], the action of nearby periodic orbits is compared to prove the existence of points outside the Mather set.

The plan of the paper is as follows: in Section 2 we review the necessary background from Aubry-Mather theory and viscosity solutions. In Section 3 we prove explicit estimates for viscosity solutions. In Section 4 we prove our main result, which gives criteria for the failure of Hamilton-Jacobi integrability. Explicit examples are discussed in Section 5. Finally, numerical results are presented in Section 6. In the remainder of this section we present our main result.

Let $L(x, v)$, the Lagrangian, be the Legendre transform of the Hamiltonian, $H(p, x)$, defined by

$$
L(x, v)=\sup _{p}-v \cdot p-H(p, x) .
$$

We assume that the Lagrangian has the form

$$
L(x, v)=\frac{1}{2} g_{i j}(x) v_{i} v_{j}+h_{i}(x) v_{i}-V(x),
$$

in which $g_{i j}(x)$ is a positive definite metric, $h_{i}$ represents the magnetic field and $V(x)$ is the potential energy. This choice of Lagrangians is quite general, as many important examples have the form (1.6). Most of what we will discuss in this paper is true in a much more general setting, but we have chosen to lose some generality in order to simplify the exposition.

We assume the following bounds for the metric $g_{i j}$, the magnetic field $h_{i}$ and potential $V$ :

(1) as a matrix, $c_{1} \leq g_{i j}(x) \leq c_{2}$, furthermore $\left|D_{x} g_{i j}(x)\right| \leq c_{3}$, and $\left|D_{x x}^{2} g_{i j}(x)\right| \leq c_{4}$;

(2) $\left|h_{i}(x)\right| \leq c_{5},\left|D_{x} h_{i}(x)\right| \leq c_{6}$, and $\left|D_{x x}^{2} h_{i}(x)\right| \leq c_{7}$;

(3) $0 \leq V(x) \leq c_{8},\left|D_{x} V\right| \leq c_{9},\left|D_{x x}^{2} V\right| \leq c_{10}$.

The Hamiltonian corresponding to $L$ is given by

$$
H(p, x)=\frac{1}{2} g^{i j}(x)\left(p_{j}-h_{j}\right)\left(p_{i}-h_{i}\right)+V(x),
$$

in which $g^{i j}$ is the inverse of $g_{i j}$, that is $g^{i k} g_{k j}=\delta_{j}^{i}$.

The main explicit estimates for viscosity solutions are summarized in the following theorem. This theorem gives a bound on the action of the orbits which start on the graphs of the Hamilton-Jacobi solutions, and therefore it is possible to use it to detect the existence of points in phase space lying outside the graph of any solution of a Hamilton-Jacobi equation.

Theorem 1.2. Let $(p, x) \in \mathbb{R}^{n} \times \mathbb{T}^{n}$ and suppose that there exists $P \in \mathbb{R}^{n}$, and a corresponding viscosity solution $u$ of (1.2) such that $p=P+D_{x} u(x)$. Let $\mathbf{x}(\cdot)$ be the solution to (1.1) with initial conditions $(p, x)$. Define

and

$$
S_{P}(t)=\int_{0}^{t} L(\mathbf{x}, \dot{\mathbf{x}})+P \dot{\mathbf{x}}+\bar{H}(P) \mathrm{d} s,
$$

Let

$$
E=H(p, x)
$$

$$
C_{0}=\frac{n}{4} \sqrt{c_{2}}\left[\left(\frac{c_{4}}{4}+4 c_{3}\right)\left(\frac{2 E}{c_{1}}+\frac{n}{2 c_{2}}\right)+c_{7}\left(\frac{2 E}{c_{1}}+\frac{n}{2 c_{2}}\right)^{1 / 2}+\frac{2 c_{6} n}{c_{2}}+\frac{c_{10}}{3}\right]^{1 / 2}
$$


and

$$
C_{1}=2 \sqrt{c_{2}}\left[\left(\frac{c_{4}}{4}+4 c_{3}\right)\left(\frac{2 E}{c_{1}}+\frac{9 n}{c_{2}}\right)+c_{7}\left(\frac{2 E}{c_{1}}+\frac{9 n}{c_{2}}\right)^{1 / 2}+\frac{4 c_{6} 9 n}{c_{2}}+\frac{c_{10}}{3}\right]^{1 / 2} c_{n},
$$

where $c_{n}$ only depends on the dimension $n$.

Then

$$
\begin{gathered}
\sup _{t}\left|S_{P}\right| \leq C_{0}, \\
\sup _{t} \frac{\left|S_{P}(t)\right|}{\|\mathbf{x}(t)-\mathbf{x}(0)\|} \leq C_{1} .
\end{gathered}
$$

Furthermore, if $u$ is $C^{2}$, let

$$
C_{2}=2 \sqrt{c_{2}}\left[\left(\frac{c_{4}}{4}+4 c_{3}\right)\left(\frac{2 E}{c_{1}}+\frac{n}{2 c_{2}}\right)+c_{7}\left(\frac{2 E}{c_{1}}+\frac{n}{2 c_{2}}\right)^{1 / 2}+\frac{2 c_{6} n}{c_{2}}+\frac{c_{10}}{3}\right]^{1 / 2} .
$$

Then

$$
\sup _{t} \sup _{k \in \mathbb{Z}^{n}} \frac{\left|S_{P}(t)-\left(D_{v} L(\mathbf{x}(0), \dot{\mathbf{x}}(0))+P\right)(\mathbf{x}(t)-\mathbf{x}(0)+k)\right|}{|\mathbf{x}(t)-\mathbf{x}(0)+k|^{2}} \leq C_{2} .
$$

Since, in principle, $P$ is unknown, the next corollary yields three criteria to prove the failure of HamiltonJacobi integrability.

Corollary 1.1. Let $(p, x) \in \mathbb{R}^{n} \times \mathbb{T}^{n}$ and suppose that there exists $P \in \mathbb{R}^{n}$, and a corresponding viscosity solution $u$ of (1.2) such that $p=P+D_{x} u(x)$. Let $\mathbf{x}(\cdot)$ be the solution to (1.1) with initial conditions $(p, x)$. Then:

$$
\begin{gathered}
\inf _{P} \sup _{t}\left|S_{P}(t)\right| \leq C_{0}, \\
\inf _{P} \sup _{t} \frac{\left|S_{P}(t)\right|}{\|\mathbf{x}(t)-\mathbf{x}(0)\|} \leq C_{1} .
\end{gathered}
$$

Furthermore, if the solution $u$ is $C^{2}$ then

$$
\inf _{P} \sup _{t} \sup _{k \in \mathbb{Z}^{n}} \frac{\left|S_{P}(t)-D_{v} L(\mathbf{x}(0), \dot{\mathbf{x}}(0))(\mathbf{x}(t)-x(0)+k)\right|}{|\mathbf{x}(t)-\mathbf{x}(0)+k|^{2}} \leq C_{2} .
$$

One might imagine that (1.10)-(1.12) could always be satisfied, however as we will argue in Section 4 this is not the case, and, as our examples illustrate, this is indeed a useful criteria for the failure of Hamilton-Jacobi integrability.

\section{MATHER MEASURES AND Viscosity SOLUtions}

In this section we summarize some background material from Mather measures and viscosity solutions. A self contained exposition of part of the material presented here can be found in the survey paper [5]. In Mather theory [31-35], instead of looking for invariant tori, one looks for probability measures $\mu$, the Mather measures, on $\mathbb{T}^{n} \times \mathbb{R}^{n}$ which minimize the action

$$
\int L(x, v)+P \cdot v \mathrm{~d} \mu
$$

and satisfy a holonomy condition:

$$
\int v D_{x} \phi \mathrm{d} \mu=0
$$

for all $\phi(x) \in C^{1}\left(\mathbb{T}^{n}\right)$. The supports of these measures are the Mather sets, and are the natural generalizations of invariant tori (and in fact agree with invariant tori if they exist). Recent results [2,6,8-13], and [19] show 
that viscosity solutions of (1.2) encode many properties of Mather sets. In particular, if $\mu$ is a Mather measure and $u$ solves (1.2) then (Thm. 22 in [5])

$$
\int L(x, v)+P \cdot v \mathrm{~d} \mu=-\bar{H}(P) .
$$

Furthermore, the support of the Mather measure is a subset of the graph (see, for instance, Thm. 30 in [5])

$$
(x, v)=\left(x,-D_{p} H\left(P+D_{x} u, x\right)\right),
$$

for any viscosity solution of (1.2). Finally, a result due to Mañé [32] (see also [14]) shows that the Mather set (and also any Mather measure) is invariant under the flow generated by the Euler-Lagrange equations (see, for instance, Thm. 43 in [5])

$$
\frac{\mathrm{d}}{\mathrm{d} t} D_{v} L(\mathbf{x}, \dot{\mathbf{x}})-D_{x} L(\mathbf{x}, \dot{\mathbf{x}})=0
$$

which is equivalent, by the Legendre transform $\mathbf{p}=-D_{v} L(\mathbf{x}, \dot{\mathbf{x}})$, to (1.1).

In the Mather set, the asymptotic behavior of the Hamiltonian dynamics is controlled by viscosity solutions. Indeed, let $(x, p)$ be any point in $\mathbb{T}^{n} \times \mathbb{R}^{n}$. Consider the solution $(\mathbf{x}, \mathbf{p})$ of the Hamilton equations (1.1) with initial condition $(x, p)$. If the point $(x, p)$ is a generic point of an ergodic component of a Mather set then

$$
\frac{\mathbf{x}(T)}{T} \rightarrow Q
$$

as $T \rightarrow \infty$, for some vector $Q \in \mathbb{R}^{n}$. Furthermore, $Q \in-\partial_{P}^{-} \bar{H}(P)$, where $\partial_{P}^{-}$denotes the subdifferential (see Thm. 1 in [19]). Thus $Q=-D_{P} \bar{H}(P)$ if $\bar{H}$ is differentiable at $P$. Furthermore, as $T \rightarrow \infty$

$$
\frac{1}{T} \int_{0}^{T} L(\mathbf{x}, \dot{\mathbf{x}}) \mathrm{d} t \rightarrow \bar{L}(Q),
$$

where $\bar{L}(Q)$ is the infimum of the average action over all trajectories with rotation number $Q$, and, additionally, $\bar{L}(Q)=-P Q-\bar{H}(P)$ is the Legendre transform of $\bar{H}(P)$.

\section{EXPlicit ESTIMATES FOR VISCOSity SOLUTIONS}

In this paper we need several explicit estimates for viscosity solutions of (1.2). These are standard in the theory, however, as we need sharp and explicit estimates, in this section we present detailed proofs.

Our framework is the following: we are given a point $(x, p) \in \mathbb{T}^{n} \times \mathbb{R}^{n}$, and we would like to investigate whether there is a vector $P$, and a viscosity solution $u$ to (1.2) such that $p=P+D_{x} u(x)$. The main difficulty with this approach is that $P$ and $u$, if exist, are unknown. Our objective in this section is to prove estimates for $P$ and $u$ that only depend on the point $(x, p)$ and other known quantities. These estimates are: bounds for second derivatives and Lipschitz constant of $u$, bounds for $P$ in terms of the initial energy, and error estimates for the numerical computation of $\bar{H}(P)$.

Let $(x, p) \in \mathbb{T}^{n} \times \mathbb{R}^{n}$ be fixed, and define $v \in \mathbb{R}^{n}$ by the Legendre transform, $p=-D_{v} L(x, v)$. The energy

$$
E(x, v)=\frac{1}{2} g_{i j}(x) v_{i} v_{j}+V(x)
$$

is conserved by the Euler-Lagrange equations. Note that the energy does not depend on the magnetic field $h$. The proof of this fact can be found in any book on classical mechanics, for instance [18].

Furthermore, along solutions of (1.1)

for $\mathbf{p}_{j}=-g_{i j} \dot{\mathbf{x}}_{i}-h_{j}$.

$$
H(\mathbf{p}, \mathbf{x})=E(\mathbf{x}, \dot{\mathbf{x}})
$$


Proposition 3.1. Let $\mathbf{x}$ be a solution to (2.3). Then

$$
|\dot{\mathbf{x}}| \leq \frac{(2 E)^{1 / 2}}{c_{1}^{1 / 2}} .
$$

Proof. Since $V \geq 0$,

$$
\frac{1}{2} c_{1}|\dot{\mathbf{x}}|^{2} \leq \frac{1}{2} g_{i j} \dot{\mathbf{x}}_{i} \dot{\mathbf{x}}_{j} \leq E .
$$

Since the energy only depends on the initial conditions, the previous result implies that one can obtain bounds on the velocity for all time that depend only on the initial conditions.

Viscosity solutions of (1.2) have an interpretation in terms of control theory: a function $u$ is a viscosity solution of (1.2) if and only if it satisfies the following fixed point identity:

$$
u(x)=\inf _{\mathbf{x}(\cdot)} \int_{0}^{t} L(\mathbf{x}, \dot{\mathbf{x}})+P \cdot \dot{\mathbf{x}}+\bar{H}(P) \mathrm{d} t+u(\mathbf{x}(t)),
$$

in which the infimum is taken over Lipschitz trajectories $\mathbf{x}(\cdot)$, with initial condition $\mathbf{x}(0)=x$.

The next proposition establishes an estimate for $P$ :

Proposition 3.2. Let

Then

$$
C=\sup _{|\omega|=1}\left|\int_{\mathbb{T}^{n}} L(x, \omega) \mathrm{d} x\right|
$$

$$
|P| \leq \bar{H}(P)+C .
$$

Remark 3.1. Since $\bar{H}(P)$ coincides with the energy $E$ given by (3.1), this yields an a-priori bound for $P$ in terms of the initial data.

Proof. From (3.2) we have

$$
-\bar{H}(P) \leq \lim _{T \rightarrow \infty} \frac{1}{T} \int_{0}^{T} L(\mathbf{x}, \dot{\mathbf{x}})+P \dot{\mathbf{x}},
$$

for any trajectory $\mathbf{x}(\cdot)$. Let $\omega$ be an arbitrary vector such that $|\omega|=1$, and $\omega \cdot k=0$ for $k \in \mathbb{Z}^{n}$ implies $k=0$, that is, the flow $\dot{\mathbf{x}}=\omega$ is ergodic on the torus $\mathbb{T}^{n}$. Let $\mathbf{x}(t)=\omega t$. Then

$$
\bar{H}(P) \geq \lim _{T \rightarrow \infty} \frac{1}{T} \int_{0}^{T} L(\mathbf{x}, \dot{\mathbf{x}})+P \cdot \dot{\mathbf{x}}=P \cdot \omega+\int_{\mathbb{T}^{n}} L(x, \omega) \mathrm{d} x .
$$

This yields:

$$
|P| \leq \bar{H}(P)+\sup _{|\omega|=1}\left|\int_{\mathbb{T}^{n}} L(x, \omega) \mathrm{d} x\right| .
$$

The next objective is to study the regularity of $u$.

Proposition 3.3. Let $u$ be a viscosity solution of (1.2). Let $k_{1} \geq 0$, and

$$
C\left(k_{1}\right)=2 \sqrt{c_{2}}\left[\left(\frac{c_{4}}{4}+4 c_{3}\right)\left(\frac{2 \bar{H}}{c_{1}}+\frac{k_{1}^{2}}{c_{2}}\right)+c_{7}\left(\frac{2 \bar{H}}{c_{1}}+\frac{k_{1}^{2}}{c_{2}}\right)^{1 / 2}+\frac{4 c_{6} k_{1}^{2}}{c_{2}}+\frac{c_{10}}{3}\right]^{1 / 2} .
$$

Then $u$ is a locally semiconcave function in $x$ with semiconcavity modulus $C\left(k_{1}\right)$ that is

$$
u(x+y)-2 u(x)+u(x-y) \leq C\left(k_{1}\right)|y|^{2},
$$

for all $|y| \leq k_{1}$. 
Remark 3.2. The main point of this lemma is not the semiconcavity per se, but the estimate for the constant $C\left(k_{1}\right)$ which is explicit in terms of the Lagrangian and the energy of an optimal trajectory.

Remark 3.3. The constant in the lemma can be bounded uniformly in $y$ for bounded values of $|y|$, as for large values of $|y|$ one can use the fact that $u$ is periodic to get a better estimate. In fact we can obtain a global bound for the semiconcavity constant in the periodic setting if we take $k_{1}=\frac{\sqrt{n}}{2}$.

Proof. Fix $x \in \mathbb{R}^{n}$, and choose any $y \in \mathbb{R}^{n}$. We claim that

$$
u(x+y)+u(x-y) \leq 2 u(x)+C|y|^{2},
$$

for the constant $C$ given in the statement.

Let $\mathbf{x}(\cdot), \mathbf{x}(0)=x$, be an optimal trajectory for $u(x)$. Clearly, for any trajectory $\mathbf{y}(\cdot)$ such that $\mathbf{y}(0)=y$

$u(x+y)+u(x-y)-2 u(x) \leq \int_{0}^{t}[L(\mathbf{x}(s)+\mathbf{y}(s), \dot{\mathbf{x}}(s)+\dot{\mathbf{y}}(s))+L(\mathbf{x}(s)-\mathbf{y}(s), \dot{\mathbf{x}}(s)-\dot{\mathbf{y}}(s))-2 L(\mathbf{x}(s), \dot{\mathbf{x}}(s))] \mathrm{d} s$.

Define $\mathbf{y}(s)=y \frac{t-s}{t}$. Observe that

$$
L(\mathbf{x} \pm \mathbf{y}, \dot{\mathbf{x}} \pm \dot{\mathbf{y}}) \leq L(\mathbf{x}, \dot{\mathbf{x}} \pm \dot{\mathbf{y}}) \pm D_{x} L(\mathbf{x}, \dot{\mathbf{x}} \pm \dot{\mathbf{y}}) y+C_{0}|y|^{2}
$$

The constant $C_{0}$ has three contributions: one comes from the bounds for the metric $g_{i j}$

$$
\begin{aligned}
\frac{1}{2} g_{i j}(\mathbf{x}+\mathbf{y})\left(\dot{\mathbf{x}}_{i}+\dot{\mathbf{y}}_{i}\right)\left(\dot{\mathbf{x}}_{j}+\dot{\mathbf{y}}_{j}\right)-\frac{1}{2} g_{i j}(\mathbf{x})\left(\dot{\mathbf{x}}_{i}+\dot{\mathbf{y}}_{i}\right)\left(\dot{\mathbf{x}}_{j}+\dot{\mathbf{y}}_{j}\right) \leq \\
\quad \frac{1}{2} D_{x} g_{i j}(\mathbf{x})\left(\dot{\mathbf{x}}_{i}+\dot{\mathbf{y}}_{i}\right)\left(\dot{\mathbf{x}}_{j}+\dot{\mathbf{y}}_{j}\right) \mathbf{y}+\frac{1}{8}\left\|D_{x x}^{2} g_{i j}\right\|_{\infty}\left(\left|\dot{\mathbf{x}}_{i}\right|^{2}+|\dot{\mathbf{y}}|^{2}\right)|y|^{2}
\end{aligned}
$$

the second one corresponds to the magnetic field,

$$
h_{i}(\mathbf{x}+\mathbf{y})\left(\dot{\mathbf{x}}_{i}+\dot{\mathbf{y}}_{i}\right)-h_{i}(\mathbf{x})\left(\dot{\mathbf{x}}_{i}+\dot{\mathbf{y}}_{i}\right) \leq D_{x} h_{i}(\mathbf{x}) \mathbf{y}\left(\dot{\mathbf{x}}_{i}+\dot{\mathbf{y}}_{i}\right)+\frac{1}{2}\left\|D_{x x}^{2} h_{i}\right\|_{\infty}|y|^{2}\left|\dot{\mathbf{x}}_{i}+\dot{\mathbf{y}}_{i}\right|
$$

and the last one from the potential energy:

$$
V(\mathbf{x}+\mathbf{y})-V(\mathbf{x}) \leq D_{x} V(\mathbf{x}) \mathbf{y}+\frac{1}{2}\left\|D_{x x}^{2} V\right\|_{\infty}|y|^{2} \frac{(t-s)^{2}}{t^{2}}
$$

Note that in the previous estimates we used $|\mathbf{y}| \leq|y|$, to estimate the second order terms. Therefore, we have the following estimate for $C_{0}$ :

$$
C_{0} \leq\left(\frac{c_{4}}{8}+2 c_{3}\right)\left(\frac{2 \bar{H}}{c_{1}}+\frac{k_{1}^{2}}{t^{2}}\right)+\frac{c_{7}}{2}\left(\frac{2 \bar{H}}{c_{1}}+\frac{k_{1}^{2}}{t^{2}}\right)^{1 / 2}+\frac{2 c_{6} k_{1}^{2}}{t^{2}}+\frac{c_{10}}{3} .
$$

Note that the term $\frac{c_{10}}{3}$ comes from the integration in time. Also we have

$$
L(\mathbf{x}, \dot{\mathbf{x}}+\dot{\mathbf{y}}, s)+L(\mathbf{x}, \dot{\mathbf{x}}-\dot{\mathbf{y}}, s) \leq 2 L(\mathbf{x}, \dot{\mathbf{x}}, s)+c_{2}|\dot{\mathbf{y}}|^{2}
$$

Thus

Therefore

$$
L(\mathbf{x}+\mathbf{y}, \dot{\mathbf{x}}+\dot{\mathbf{y}}, s)+L(\mathbf{x}-\mathbf{y}, \dot{\mathbf{x}}-\dot{\mathbf{y}}, s) \leq 2 L(\mathbf{x}, \dot{\mathbf{x}}, s)+2 C_{0}|y|^{2}+2 c_{2} \frac{|y|^{2}}{t^{2}}
$$

$$
u(x+y)-2 u(x)+u(x-y) \leq 2 C_{0}|y|^{2} t+2 c_{2} \frac{|y|^{2}}{t}
$$


By choosing $t=\sqrt{\frac{c_{2}}{C_{0}}}$ we obtain

$$
u(x+y)-2 u(x)+u(x-y) \leq C|y|^{2}
$$

with

$$
C=2 \sqrt{c_{2} C_{0}} .
$$

As corollaries to this proposition we obtain the two following estimates for $\|u\|_{\infty}$ and its Lipschitz constant.

Proposition 3.4. Let $u$ be a solution of (1.2). Then, after adding a suitable constant to $u$, the $L^{\infty}$ norm of $u$ can be estimated by

$$
\frac{n}{8} \sqrt{c_{2}}\left[\left(\frac{c_{4}}{4}+4 c_{3}\right)\left(\frac{2 E}{c_{1}}+\frac{n}{2 c_{2}}\right)+c_{7}\left(\frac{2 E}{c_{1}}+\frac{n}{2 c_{2}}\right)^{1 / 2}+\frac{2 c_{6} n}{c_{2}}+\frac{c_{10}}{3}\right]^{1 / 2} .
$$

Proof. Suppose $u$ is a $\mathbb{Z}^{n}$ periodic semiconcave function with semiconcavity modulus $C$. Without loss of generality, suppose that $x=0$ is its minimum. Then

$$
u(y) \leq u(0)+\frac{1}{2} C|y|^{2} .
$$

By periodicity, the maximum of $u$ is achieved at a point $y$, with $|y| \leq \frac{\sqrt{n}}{2}$. Since, by adding an appropriate constant we may assume $u(y)=-u(0)$. Then we have

$$
\|u\|_{\infty} \leq \frac{C n}{16}
$$

The estimate follows from Proposition 3.3.

Proposition 3.5. Let $u$ be a solution of (1.2). Then the Lipschitz constant of $u$ is bounded by

$$
2 \sqrt{c_{2}}\left[\left(\frac{c_{4}}{4}+4 c_{3}\right)\left(\frac{2 \bar{H}}{c_{1}}+\frac{9 n}{c_{2}}\right)+c_{7}\left(\frac{2 \bar{H}}{c_{1}}+\frac{9 n}{c_{2}}\right)^{1 / 2}+\frac{4 c_{6} 9 n}{c_{2}}+\frac{c_{10}}{3}\right]^{1 / 2} c_{n},
$$

in which $c_{n}$ is a constant that depends only on the dimension $n$.

Proof. It is well known that a periodic semiconcave function $\phi$ is Lipschitz. To prove this fact, observe that

$$
D \phi(x)=D \phi(y)+\int_{0}^{1} D^{2} \phi(s x+(1-s) y)(x-y) \mathrm{d} s .
$$

Since $\phi$ is periodic, there exists $y$ such that $|x-y| \leq 3 \sqrt{n}, D \phi(y)=0$, and

$$
-\tilde{c}_{n}|D \phi(x)| \geq D \phi(x) \cdot(x-y) \geq-C|x-y|^{2},
$$

where $\tilde{c}_{n}$ is a constant that depends only on the dimension $n$. Therefore

$$
|D \phi(x)| \leq c_{n} C .
$$

Furthermore, in the estimate for the semiconcavity constant $C$ we may take $k_{1}=3 \sqrt{n}$. 
Proposition 3.6. Assume that $u$ is a smooth solution of the Hamilton-Jacobi equation (1.2). Let $k_{1} \geq 0$, and

$$
C\left(k_{1}\right)=2 \sqrt{c_{2}}\left[\left(\frac{c_{4}}{4}+4 c_{3}\right)\left(\frac{2 \bar{H}}{c_{1}}+\frac{k_{1}^{2}}{c_{2}}\right)+c_{7}\left(\frac{2 \bar{H}}{c_{1}}+\frac{k_{1}^{2}}{c_{2}}\right)^{1 / 2}+\frac{4 c_{6} k_{1}^{2}}{c_{2}}+\frac{c_{10}}{3}\right]^{1 / 2} .
$$

Then $u$ is a locally semiconvex function in $x$ with semiconcavity modulus $C\left(k_{1}\right)$ that is

$$
u(x+y)-2 u(x)+u(x-y) \geq-C\left(k_{1}\right)|y|^{2},
$$

for all $|y| \leq k_{1}$.

Remark 3.4. The main point of assuming that a solution $u$ is smooth is that, then, the method of characteristics is valid, and so for every point $x$ there is a global characteristic.

Proof. Fix $x \in \mathbb{R}^{n}$ and choose any $y \in \mathbb{R}^{n}$. We claim that

$$
u(x+y)+u(x-y) \geq 2 u(x)-C|y|^{2} .
$$

Let $\mathbf{x}(t)$ be an optimal trajectory, $x=\mathbf{x}(t)$, and $x_{0}=\mathbf{x}(0)$. Then

$$
u\left(x_{0}\right)=\int_{0}^{t}[L(\mathbf{x}(s), \dot{\mathbf{x}}(s))+P \dot{\mathbf{x}}] \mathrm{d} s+u(x) .
$$

Therefore

$$
\begin{aligned}
u(x-y)-2 u(x)+u(x-y) \geq & \int_{0}^{t}[-L(\mathbf{x}(s)+\mathbf{y}(s), \dot{\mathbf{x}}(s)+\dot{\mathbf{y}}(s)) \\
& -L(\mathbf{x}(s)+\mathbf{y}(s), \dot{\mathbf{x}}(s)+\dot{\mathbf{y}}(s))+2 L(\mathbf{x}(s), \dot{\mathbf{x}}(s))] \mathrm{d} s,
\end{aligned}
$$

if we choose $\mathbf{y}(s)=y \frac{s}{t}$. From this inequality and proceeding exactly as in Proposition 3.3 we obtain the estimate.

Given a value $P$, there are efficient numerical methods to compute $\bar{H}(P)$, and control the error [22] (for an alternative scheme, consult [36]). The algorithm in [22] is based on the representation formula for $\bar{H}(P)$

$$
\bar{H}(P)=\inf _{\phi \in C_{\mathrm{per}}^{1}} \sup _{x} H\left(P+D_{x} \phi, x\right)
$$

due to [6] (see also, for a more general setting, [21,26]).

Let $T_{h}$ be a set of piecewise linear finite elements, with the diameter of each element bounded by $h$. Let $\bar{H}_{h}(P)$ be the numerical approximation computed by:

$$
\bar{H}_{h}(P)=\inf _{\phi \in T_{h}} \operatorname{esssup} H\left(D_{x} \phi, x\right) .
$$

The main error estimate is:

Proposition 3.7. For any convex Hamiltonian $H(p, x)$ for which (1.2) has a viscosity solution

$$
\bar{H} \leq \bar{H}_{h}(P) .
$$

Furthermore, if (1.2) has a smooth viscosity solution then

$$
\bar{H}_{h}(P) \leq \bar{H}(P)+C h .
$$


The constant $C$ depends only on bounds for the Hamiltonian, the energy level, but not on $P$, and can be estimated by

in which

$$
C \leq\left\|D_{x} H(p, x)\right\|_{\infty, *}+\left\|D_{x x}^{2} u\right\|_{\infty}\left\|D_{p} H(p, x)\right\|_{\infty, *} \frac{\sqrt{n}}{2}
$$

$$
\|f(p, x)\|_{\infty, *}=\sup _{x \in \mathbb{T}^{n},|p|<R}|f(p, x)|
$$

and $R$ is a bound for $\left|P+D_{x} u\right|$.

Proof. The first claim, that is,

$$
\bar{H}=\inf _{\psi \in C^{1}\left(\mathbb{T}^{n}\right)} \sup _{x} H\left(P+D_{x} \psi, x\right) \leq \inf _{\phi \in T_{h}} \underset{x}{\operatorname{esssup}} H\left(P+D_{x} \phi, x\right),
$$

can be proved in the following way: to each $\phi \in T_{h}$ we associate a function

$$
\psi=\phi * \eta_{\epsilon} \in C^{1}\left(\mathbb{T}^{n}\right) .
$$

Then the convexity of $H$ implies

$$
\sup _{x} H\left(P+D_{x} \psi, x\right) \leq \underset{x}{\operatorname{esssup}} H\left(P+D_{x} \phi, x\right)+O(\epsilon),
$$

since

$$
H\left(P+D_{x}\left(\phi * \eta_{\epsilon}\right)(x), x\right) \leq \int H\left(P+D_{x} \phi(y), y\right) \eta_{\epsilon}(x-y) \mathrm{d} y+O(\epsilon)
$$

Since $\epsilon$ is arbitrary, we get the desired inequality.

Suppose $u$ is smooth viscosity solution. Construct a function $\phi_{u} \in T_{h}$ by interpolating linearly the values of $u$ at the nodal points. At a node $x$ we have

$$
D_{x_{j}} \phi\left(x_{i}\right)=\frac{u\left(x+h e_{j}\right)-u(x)}{h}=\int_{0}^{1} D_{x_{j}} u\left(x+s h e_{j}\right) \mathrm{d} s
$$

In each triangle $T^{i}$, the oscillation of the derivative of $u$ can be estimated by

$$
\left|D_{x} u(x)-D_{x} u(y)\right| \leq\left\|D_{x x}^{2} u\right\|_{\infty}|x-y|
$$

which implies

Thus at a node $x$ we have

$$
\left|D_{x_{j}} \phi\left(x_{i}\right)-D_{x_{j}} u(x)\right| \leq\left\|D_{x x}^{2} u\right\|_{\infty} \frac{h}{2}
$$

$$
\left|H\left(D_{x} \phi_{u}(x), x\right)-\bar{H}(P)\right| \leq\left\|D_{x x}^{2} u\right\|_{\infty}\left\|D_{p} H(p, x)\right\|_{\infty, *} \frac{h}{2} \sqrt{n} .
$$

We also have, for all points $y$ in the triangle,

$$
\left|H\left(D_{x} \phi_{u}(x), x\right)-H\left(D_{x} \phi_{u}(x), y\right)\right| \leq\left\|D_{x} H(p, x)\right\|_{\infty, *}|x-y| .
$$

Then

$$
\operatorname{esssup} H\left(P+D_{x} \phi_{u}, x\right) \leq \bar{H}(P)+\left\|D_{x} H(p, x)\right\|_{\infty, *} h+\left\|D_{x x}^{2} u\right\|_{\infty}\left\|D_{p} H(p, x)\right\|_{\infty, *} \frac{h}{2} \sqrt{n}
$$




\section{DeteCtion OF NON-INTEGRABiLity}

In this section we prove Theorem 1.2 by putting together the previous estimates.

Proof of Theorem 1.2. If $(x, v)$ belongs to the graph of a viscosity solution $u$ of $(1.2)$, and $\mathbf{x}(\cdot)$ is the corresponding solution of the Euler-Lagrange flow, then for all $t$

$$
u(x)=\int_{0}^{t} L(\mathbf{x}, \dot{\mathbf{x}})+P \dot{\mathbf{x}}+\bar{H}(P) \mathrm{d} s+u(\mathbf{x}(t)) .
$$

First, since $u$ is bounded, there exists a constant $C_{0}=2\|u\|_{\infty}$ such that

$$
\sup _{t}|u(x)-u(\mathbf{x}(t))| \leq C_{0}
$$

therefore we have (1.7).

An improved version of this estimate follows from the fact that $u$ is periodic and Lipschitz, and so if $C_{1}=$ $\|D u\|_{\infty}$ is the Lipschitz constant of $u$ then

$$
\sup _{t} \frac{|u(x)-u(\mathbf{x}(t))|}{\|\mathbf{x}(t)-\mathbf{x}(0)\|} \leq C_{1}
$$

in which $\|x-y\|=\inf _{k \in \mathbb{Z}^{n}}|x-y+k|$ is the periodic distance. Therefore we have (1.8).

Since $p=P+D_{x} u$, and $p=-D_{v} L(\mathbf{x}, \dot{\mathbf{x}})$, we have

$$
D_{x} u(\mathbf{x})=-D_{v} L(\mathbf{x}, \dot{\mathbf{x}})-P .
$$

If the solution $u$ is $C^{2}$, the previous estimate can be improved since we have a priori estimates for second derivatives

$$
\sup _{t} \sup _{k \in \mathbb{Z}^{n}} \frac{\left|u(x)+D_{x} u(x)(\mathbf{x}(t)-x+k)-u(\mathbf{x}(t)+k)\right|}{|\mathbf{x}(t)-\mathbf{x}(0)+k|^{2}} \leq C_{2},
$$

and $u(\mathbf{x}(t)+k)=u(\mathbf{x}(t))$, this yields (1.9).

To check that, for a given a point $(p, x)$, there does not exist a vector $P \in \mathbb{R}^{n}$ and a viscosity solution $u$ such that $p=P+D_{x} u(x)$, we will proceed by contradiction by checking the inequalities in Corollary 1.1 . These inequalities involve the values $P$ and $\bar{H}(P)$, both of them unknown. However, the value $\bar{H}(P)$ can be well approximated by the minimax representation formula (3.3), provided that to $P$ corresponds and invariant tori. The error of the approximation depends on the energy, which can be estimated by the initial condition $(x, v)$, but is independent of $P$. The solution of (2.3) can be computed with arbitrary precision using a suitable numerical solver. Therefore, given a number $P$, we can test the inequalities (1.10), (1.11), or (1.12). Due to the small denominators that can arise in (1.11) and (1.12), our error estimates are largely amplified and therefore we have only implemented the numerical computation of (1.10).

There is a heuristic explanation why the terminal time (or any large value of $t$ ) may be enough. Let $(x, v)$ be a generic point in an ergodic component of the Mather set, and $\mathbf{x}$ the corresponding trajectory.

If the solution $\mathbf{x}$ has rotation number $Q$ but

$$
\liminf _{T \rightarrow \infty} \frac{1}{T} \int_{0}^{T} L(\mathbf{x}, \dot{\mathbf{x}}) \mathrm{d} t>\bar{L}(Q)+\epsilon,
$$

for some $\epsilon>0$, the initial condition is not a generic point in an ergodic component of the Mather set. Therefore, proving the existence of gaps of the Mather sets. If (4.1) holds, then, since $\bar{L}(Q)-P Q-\bar{H}(P)=0$,

$$
\int_{0}^{T} L(\mathbf{x}, \dot{\mathbf{x}})+P \dot{\mathbf{x}}+\bar{H}(P) \mathrm{d} s>T \epsilon \rightarrow \infty,
$$

as $T \rightarrow \infty$. Therefore (1.10), (1.11) and (1.12) cannot be satisfied. 


\section{EXPLICIT EXAMPLES}

In this section we consider two examples: the one dimensional pendulum, and linear Hamiltonians, which can be studied explicitly.

\subsection{The one dimensional pendulum}

The Hamiltonian corresponding to a one-dimensional pendulum with mass and length normalized to be 1 , is

$$
H(p, x)=\frac{p^{2}}{2}-\cos 2 \pi x .
$$

For this Hamiltonian one can determine the solution of (1.2). Indeed, for each $P \in \mathbb{R}$, and for a.e. $x \in \mathbb{R}$, the solution $u(P, x)$ satisfies

$$
\frac{\left(P+D_{x} u\right)^{2}}{2}=\bar{H}(P)+\cos 2 \pi x .
$$

This implies $\bar{H}(P) \geq 1$, and so

$$
D_{x} u=-P \pm \sqrt{2(\bar{H}(P)+\cos 2 \pi x)}, \quad \text { for a.e. } \quad x \in \mathbb{R}
$$

Thus

$$
u=\int_{0}^{x}-P+s(y) \sqrt{2(\bar{H}(P)+\cos 2 \pi y)} \mathrm{d} y
$$

where $|s(y)|=1$. Because $H$ is convex in $p$ and $u$ is a viscosity solution, it is semiconcave. So, the only possible discontinuities in the derivative of $u$ are the ones that satisfy $D_{x} u\left(x^{-}\right)-D_{x} u\left(x^{+}\right)>0$. Therefore $s$ can change sign from 1 to -1 at any point but jumps from -1 to 1 can happen only when $\sqrt{2(\bar{H}(P)+\cos 2 \pi x)}=0$. If we require 1-periodicity there are two cases, first if $\bar{H}(P)>1$ the solution is $C^{1}$ since $\sqrt{2(\bar{H}(P)+\cos 2 \pi y)}$ is never zero. These solutions correspond to invariant tori. In this case, $P$ and $\bar{H}(P)$ satisfy the equation

$$
P= \pm \int_{0}^{1} \sqrt{2(\bar{H}(P)+\cos 2 \pi y)} \mathrm{d} y .
$$

It is easy to check that this equation has a solution $\bar{H}(P)$ whenever

$$
|P| \geq \int_{0}^{1} \sqrt{2(1+\cos 2 \pi y)} \mathrm{d} y
$$

that is

$$
|P| \geq \frac{4}{\pi} \simeq 1.27324
$$

When this inequality fails, $\bar{H}(P)=1$ and $s(x)$ can have a discontinuity. Indeed, $s(x)$ jumps from -1 to 1 when $x=\frac{1}{2}+k$, with $k \in \mathbb{Z}$, and there is a point $x_{0}$ defined by the equation

$$
-\int_{0}^{1} s(y) \sqrt{2(1+\cos 2 \pi y)} \mathrm{d} y=P,
$$

such that $s(x)$ jumps from 1 to -1 at $x_{0}+k, k \in \mathbb{Z}$.

Therefore, for initial conditions $(x, p)$ such that the energy $E<1$, there is no corresponding vector $P$ and solution $u(x)$ so that

$$
H\left(P+D_{x} u, x\right)=E,
$$


thus these energy levels should be non-integrable. In fact, we can detect this behavior using our methods. We have

$$
\begin{aligned}
S_{P}(T) & =\int_{0}^{T} L(\mathbf{x}, \dot{\mathbf{x}})+P \dot{\mathbf{x}}+\bar{H}(P) \\
& =\int_{0}^{T}(P-\mathbf{p}(t)) \dot{\mathbf{x}}(t)-H(\mathbf{p}(t), \mathbf{x}(t))+\bar{H}(P) .
\end{aligned}
$$

For $E<1$ the trajectories are periodic, thus

$$
\int_{0}^{T_{n}} P \dot{\mathbf{x}}=0
$$

for any multiple $T_{n}$ of the period. We have $\dot{\mathbf{x}}=-\mathbf{p}(t)$, therefore

$$
\int_{0}^{T_{n}}-\mathbf{p}(t) \dot{\mathbf{x}}(t)=\int_{0}^{T_{n}}|\mathbf{p}(t)|^{2} \geq 0 .
$$

Since $\bar{H}(P)-H(\mathbf{p}, \mathbf{x}) \geq 1-E>0$, the integral

$$
\int_{0}^{T_{n}} \bar{H}(P)-H(\mathbf{p}(t), \mathbf{x}(t)) \mathrm{d} t
$$

is unbounded.

\subsection{Linear Hamiltonians}

It is well known that there may not exist smooth solutions to the linear Hamilton-Jacobi equation

$$
\omega \cdot\left(P+D_{x} u\right)+V(x)=\bar{H}(P)
$$

with $\omega \in \mathbb{R}^{n}$. The Hamiltonian $H(p, x)=\omega \cdot p+V(x)$ is convex in $p$ but not strictly convex. However as we will show, our methods still detect non-integrability in some cases.

The identity

$$
\int \omega \cdot\left(P+D_{x} u\right)+V(x)=\int \bar{H}(P)
$$

is a necessary condition for the existence of solutions of the Hamilton-Jacobi equation. Thus

$$
\bar{H}(P)=\omega \cdot P+\bar{H}(0),
$$

with $\bar{H}(0)=\int V(x)$, which we can assume to be zero.

The Lagrangian corresponding to the Hamiltonian is

$$
L(v, x)= \begin{cases}-V(x) & \text { if } v=-\omega \\ +\infty & \text { otherwise }\end{cases}
$$

The equation of the dynamics are

$$
\begin{aligned}
& \dot{\mathbf{x}}=-\omega \\
& \dot{\mathbf{p}}=-2 \pi \sin \left(2 \pi \mathbf{x}_{1}\right)
\end{aligned}
$$


Therefore, the action $S_{P}(T)$ is given by

$$
S_{P}(T)=\int_{0}^{T}-V(\mathbf{x}(t)) \mathrm{d} t
$$

with $\mathbf{x}(t)=\mathbf{x}(0)+\omega t$.

We consider three examples. The first one:

$$
H(p, s)=(1, \sqrt{2}) \cdot p+\cos \left(2 \pi x_{1}\right)
$$

that is, $\omega=(1, \sqrt{2})$. In this case, one can construct a smooth solution to the Hamilton-Jacobi equation. Therefore

$$
S_{P}(T)=\int_{0}^{T}-\cos \left(2 \pi \mathbf{x}_{1}(0)+2 \pi t\right) \mathrm{d} t
$$

is bounded uniformly in $T$ for any value of $\mathbf{x}_{1}(0)$, which can be checked directly.

The second case is $\omega=(1,1)$, and the equation reads

$$
(1,1) \cdot D u+\cos \left(2 \pi x_{1}\right)=0 .
$$

This is a resonant example since $(1,1)$ is rationally dependent. However one can still find a smooth solution to the Hamilton-Jacobi equations by using Fourier series. The action

$$
S_{P}(T)=\int_{0}^{T}-\cos \left(2 \pi \mathbf{x}_{1}(0)+2 \pi t\right) \mathrm{d} t
$$

is again bounded uniformly in $T$ for any value of $x_{1}(0)$.

Resonant linear Hamiltonians as the previous one may fail to have a viscosity solution. An example is

$$
(0,1) \cdot D u+\sin \left(2 \pi x_{1}\right)=\bar{H}
$$

The variational formula yields

$$
\bar{H}(0)=\inf _{\phi} \sup _{x} H(D \phi, x)=1 .
$$

which is a contradiction. And, in fact, the action is

$$
S_{P}(T)=\int_{0}^{T} 1+\sin \left(2 \pi \mathbf{x}_{1}(0)\right) \mathrm{d} t,
$$

which is unbounded in $T$ for $\mathbf{x}_{1}(0) \neq \frac{3}{4}$.

\section{Computational Examples}

In this section we consider several Hamiltonian systems and try to study their integrability numerically. The first example which fits directly into our framework, are two coupled penduli. In the last example, a forced pendulum, our estimates do not apply directly, but can easily be modified so that we can also study its non-integrable regions.

To compute numerically $\bar{H}$ we have used the numerical implementation of the minimax formula (3.3) in [22], using recursive mesh refinement for speed up. To implement the ODE's we used MATLAB's solver, and used energy conservation to verify accuracy. Alternatively one could use other integrators, such as symplectic ODE 


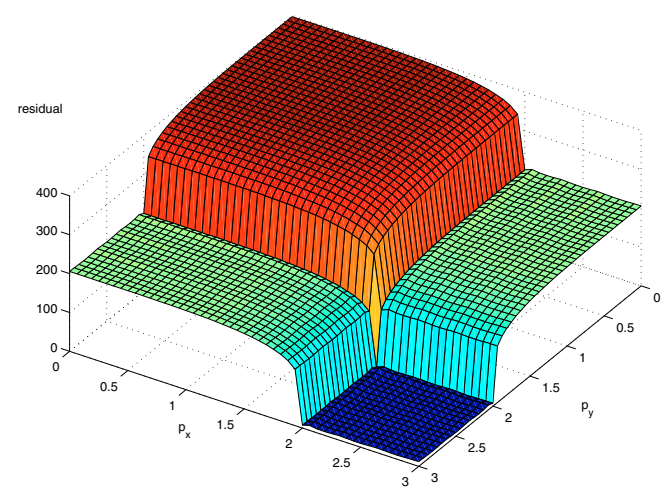

FiguRE 1. $\inf _{P} S_{P}(100)$ for coupled pendulum (no coupling, $\epsilon=0.0$ ).

solvers, however the solution of the ODE by MATLAB is quite accurate for our purposes. We only consider the first method to detect non-integrability, and plot for $T=100$ the value

$$
\inf _{P} S_{P}(T)
$$

\subsection{Coupled penduli}

The Hamiltonian for two coupled penduli is given by

$$
H\left(p_{x}, p_{y}, x, y\right)=\frac{p_{x}^{2}+p_{y}^{2}}{2}+\cos 2 \pi x+\cos 2 \pi y+\epsilon \cos 2 \pi(x-y),
$$

and the corresponding Lagrangian is

$$
L\left(v_{x}, v_{y}, x, y\right)=\frac{v_{x}^{2}+v_{y}^{2}}{2}-\cos 2 \pi x-\cos 2 \pi y-\epsilon \cos 2 \pi(x-y) .
$$

The equations of the dynamics are

$$
\begin{aligned}
\dot{\mathbf{p}}_{x} & =-2 \pi \sin 2 \pi \mathbf{x}-2 \pi \epsilon \sin 2 \pi(\mathbf{x}-\mathbf{y}) \\
\dot{\mathbf{p}}_{y} & =-2 \pi \sin 2 \pi \mathbf{y}+2 \pi \epsilon \sin 2 \pi(\mathbf{x}-\mathbf{y}) \\
\dot{\mathbf{x}} & =-\mathbf{p}_{x} \\
\dot{\mathbf{y}} & =-\mathbf{p}_{y} .
\end{aligned}
$$

To plot the non integrable regions we choose an initial point $(x, y)=\left(\frac{1}{2}, \frac{1}{2}\right)$ and then vary the values of $p_{x}$ and $p_{y}$. To compute $\bar{H}$ we have used a $12 \times 12$ grid.

The case with no coupling, $\epsilon=0$, Figure 1 is simply a two dimensional version of the pendulum example. In this case, one should note that $(x, y)=\left(\frac{1}{2}, \frac{1}{2}\right)$ and $p_{x}=p_{y}=2$ corresponds exactly to the separatrices of the (uncoupled) penduli since the energy level is $H=2$.

When coupling is positive, $\epsilon=0.2$ in Figure 2, there is numerical evidence of resonances between the center equilibrium in one pendulum and periodic orbits in the other. Note that this break up occurs close to the separatrices of the penduli, as one would expect. 


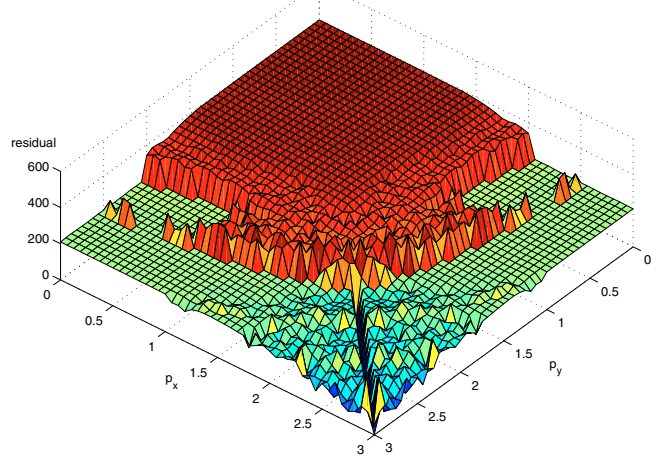

Figure 2. $\inf _{P} S_{P}(100)$ for coupled pendulum $(\epsilon=0.2)$.

\section{Error estimates}

For $\epsilon \leq 0.2$, the second derivative of the potential $\left\|D^{2} V\right\|_{\infty} \leq 55.27$. This yields a bound for $\left\|D_{x x}^{2} u\right\|_{\infty} \leq 8.58$, which implies the $L^{\infty}$ bound for $u$

$$
\|u\|_{\infty} \leq 1.07
$$

Furthermore, we have $\left\|D_{x} H\right\|_{\infty} \leq 10.65$, and for the initial conditions we consider $\left(0 \leq p_{x}, p_{y} \leq 3\right)$ we have $\left\|D_{p} H\right\| \leq 4.24$.

With a grid of $12 \times 12$ nodes $h=\frac{1}{12}$. Thus the error term in the computation of $\bar{H}$ is bounded by 3.03.

This means that values of $\inf _{P} S_{P}(100)$ over 305.15 indicate that the system is non Hamilton-Jacobi integrable. Note that these bounds are really coarse, as the error in computing $\bar{H}$ is in fact quite smaller than our estimates [22].

\subsection{Time-periodic Hamiltonians}

Our last example is a periodic time-dependent, one space dimension Hamilton-Jacobi equation:

$$
-u_{t}+H\left(D_{x} u, x, t\right)=\bar{H}
$$

There exists a unique value $\bar{H}$ for which this problem admits space-time periodic viscosity solutions, see for instance [9]. Moreover this solution is Lipschitz.

Note also that $P=\left(P_{t}, P_{x}\right)$ but $\bar{H}(P)$ is linear in $P_{t}$ so we may as well consider just the problem

$$
\inf _{\phi} \sup _{(x, t)}-\phi_{t}+H\left(P_{x}+D_{x} \phi, x, t\right)=\bar{H}\left(P_{x}\right) .
$$

This problem is not exactly in the form discussed previously in the paper. The previous estimates could be adapted, although we do not do it here for the sake of simplicity. In this example we set up a forced pendulum with time-dependent Hamiltonian

$$
H(p, x)=\frac{p^{2}}{2}+(1+\epsilon \sin 2 \pi t) \cos 2 \pi x .
$$

The equations of motion are

$$
\begin{aligned}
& \dot{\mathbf{p}}=-2 \pi(1+\epsilon \cos 2 \pi t) \sin 2 \pi \mathbf{x} \\
& \dot{\mathbf{x}}=-\mathbf{p} .
\end{aligned}
$$




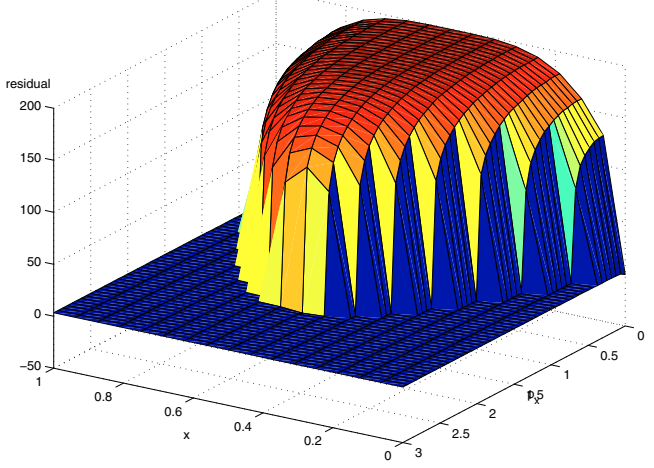

Figure $3 . \inf _{P} S_{P}(100)$ for forced pendulum (no forcing, $\epsilon=0$ ).

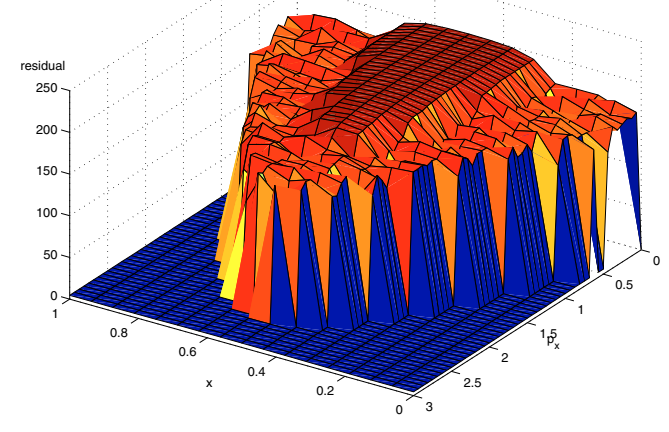

Figure $4 . \inf _{P} S_{P}(100)$ for forced pendulum $(\epsilon=0.4)$.

The corresponding Lagrangian is

$$
L(x, v, t)=\frac{v^{2}}{2}-\cos 2 \pi x-\epsilon \cos 2 \pi x \cos 2 \pi t
$$

Figures 3 and 4 show, from no forcing $(\epsilon=0)$ to large forcing $(\epsilon=0.4)$ the evolution of the heteroclinic region of the pendulum and show its break-up.

\section{Conclusions}

In this paper we have developed a set of necessary conditions for Hamilton-Jacobi integrability. These conditions can be easily implemented numerically and the error terms coming from the discretization can be estimated explicitly. Therefore the numerical results provide a rigorous proof of non Hamilton-Jacobi integrability. Both in explicit examples, as well as in more complex cases, we are able to detect behaviors such as resonances and heteroclinic break-up. The main numerical problems are due to the fact that the estimates for the error in the numerical computation for $\bar{H}$ overestimate the error, in fact, as the simulations in [22] show, the errors are quite small. To sum up, we believe that these methods are an effective way to study in practice the integrability of Hamiltonian systems. 


\section{REFERENCES}

[1] V.I. Arnold, V.V. Kozlov and A.I. Neishtadt, Mathematical aspects of classical and celestial mechanics. Springer-Verlag, Berlin (1997). Translated from the 1985 Russian original by A. Iacob, reprint of the original English edition from the series Encyclopaedia of Mathematical Sciences [Dynamical systems III, Encyclopaedia Math. Sci. 3, Springer, Berlin (1993) MR 95d:58043a].

[2] W.E. Aubry, Mather theory and periodic solutions of the forced Burgers equation. Comm. Pure Appl. Math. 52 (1999) $811-828$.

[3] M. Bardi and I. Capuzzo-Dolcetta, Optimal control and viscosity solutions of Hamilton-Jacobi-Bellman equations. Birkhäuser Boston Inc., Boston, MA, USA (1997).

[4] U. Bessi, An analytic counterexample to the KAM theorem. Ergod. Theory Dyn. Syst. 20 (2000) 317-333.

[5] A. Biryuk and D. Gomes, An introduction to the Aubry-Mather theory. São Paulo Journal of Mathematical Sciences (to appear).

[6] G. Contreras, R. Iturriaga, G.P. Paternain and M. Paternain, Lagrangian graphs, minimizing measures and Mañé's critical values. Geom. Funct. Anal. 8 (1998) 788-809.

[7] L.C. Evans, Partial differential equations. American Mathematical Society, Providence, RI, USA (1998).

[8] L.C. Evans and D. Gomes, Effective Hamiltonians and averaging for Hamiltonian dynamics. I. Arch. Ration. Mech. Anal. 157 (2001) $1-33$

[9] L.C. Evans and D. Gomes, Effective Hamiltonians and averaging for Hamiltonian dynamics. II. Arch. Ration. Mech. Anal. 161 (2002) 271-305.

[10] A. Fathi, Solutions KAM faibles conjuguées et barrières de Peierls. C. R. Acad. Sci. Paris Sér. I Math. 325 (1997) 649-652.

[11] A. Fathi, Théorème KAM faible et théorie de Mather sur les systèmes lagrangiens. C. R. Acad. Sci. Paris Sér. I Math. 324 (1997) 1043-1046.

[12] A. Fathi, Orbite hétéroclines et ensemble de Peierls. C. R. Acad. Sci. Paris Sér. I Math. 326 (1998) 1213-1216.

[13] A. Fathi, Sur la convergence du semi-groupe de Lax-Oleinik. C. R. Acad. Sci. Paris Sér. I Math. 327 (1998) 267-270.

[14] A. Fathi and A. Siconolfi, Existence of $C^{1}$ critical subsolutions of the Hamilton-Jacobi equation. Invent. Math. 155 (2004) $363-388$.

[15] W.H. Fleming and H.M. Soner, Controlled Markov processes and viscosity solutions. Springer-Verlag, New York (1993).

[16] G. Forni, Analytic destruction of invariant circles. Ergod. Theory Dyn. Syst. 14 (1994) 267-298.

[17] G. Forni, Construction of invariant measures supported within the gaps of Aubry-Mather sets. Ergod. Theory Dyn. Syst. 16 (1996) 51-86.

[18] H. Goldstein, Classical mechanics. Addison-Wesley Publishing Co., Reading, Mass., second edition (1980).

[19] D.A. Gomes, Viscosity solutions of Hamilton-Jacobi equations and asymptotics for Hamiltonian systems. Calc. Var. Partial Differential Equations 14 (2002) 345-357.

[20] D.A. Gomes, Perturbation theory for viscosity solutions of Hamilton-Jacobi equations and stability of Aubry-Mather sets. SIAM J. Math. Anal. 35 (2003) 135-147 (electronic).

[21] D.A. Gomes, Duality principles for fully nonlinear elliptic equations, in Trends in partial differential equations of mathematical physics, Progr. Nonlinear Differential Equations Appl. 61, Birkhäuser, Basel (2005) 125-136.

[22] D.A. Gomes and A.M. Oberman, Computing the effective Hamiltonian using a variational approach. SIAM J. Contr. Opt. 43 (2004) 792-812 (electronic).

[23] D.A. Gomes and E. Valdinoci, Lack of integrability via viscosity solution methods. Indiana Univ. Math. J. 53 (2004) $1055-1071$.

[24] À. Haro, Converse KAM theory for monotone positive symplectomorphisms. Nonlinearity 12 (1999) 1299-1322.

[25] A. Knauf, Closed orbits and converse KAM theory. Nonlinearity 3 (1990) 961-973.

[26] P.L. Lions and P. Souganidis, Correctors for the homogenization of Hamilton-Jacobi equations in the stationary ergodic setting. Comm. Pure Math. Appl. 56 (2003) 1501-1524.

[27] P.L. Lions, G. Papanicolao and S.R.S. Varadhan, Homogeneization of Hamilton-Jacobi equations. Preliminary version (1988).

[28] R.S. MacKay, Converse KAM theory, in Singular behavior and nonlinear dynamics, Vol. 1 (Sámos, 1988), World Sci. Publishing, Teaneck, USA (1989) 109-113.

[29] R.S. MacKay and I.C. Percival, Converse KAM: theory and practice. Comm. Math. Phys. 98 (1985) 469-512.

[30] R.S. MacKay, J.D. Meiss and J. Stark, Converse KAM theory for symplectic twist maps. Nonlinearity 2 (1989) 555-570.

[31] R. Mañé, On the minimizing measures of Lagrangian dynamical systems. Nonlinearity 5 (1992) 623-638.

[32] R. Mañé, Generic properties and problems of minimizing measures of Lagrangian systems. Nonlinearity 9 (1996) 273-310.

[33] J.N. Mather, Minimal action measures for positive-definite Lagrangian systems, in IXth International Congress on Mathematical Physics (Swansea, 1988), Hilger, Bristol (1989) 466-468.

[34] J.N. Mather, Minimal measures. Comment. Math. Helv. 64 (1989) 375-394.

[35] J.N. Mather, Action minimizing invariant measures for positive definite Lagrangian systems. Math. Z. 207 (1991) 169-207.

[36] J. Qian, Two approximations for effective hamiltonians arising from homogenization of Hamilton-Jacobi equations. Preprint (2003). 